\title{
ANÁLISIS DE OBLIGACIONES FISCALES ESTABLECIDAS PARA ASOCIACIONES DE ÁMBITO SOCIOECONÓMICO EN JIPIJAPA, MANABÍ
}

\author{
AUTORES: William Patricio Proaño Ponce ${ }^{1}$ \\ Tamara Valeria Alay Barcia ${ }^{2}$
}

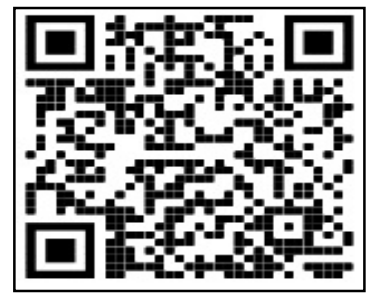

\section{DIRECCIÓN PARA CORRESPONDENCIA: mantawilliam@gmail.com}

Fecha de recepción: 17/09/2020

Fecha de aceptación: 25/11/2020

\section{RESUMEN}

En la actualidad, cumplir con deberes tributarios se ha vuelto un derecho para toda sociedad. Sin embargo, muchas asociaciones de ámbito socioeconómico en Ecuador no se encuentran informadas en su totalidad sobre los derechos que corresponden como tal. Entre los factores están la desinformación y la exigua cultura tributaria. Es por ello que resulta fundamental que los comerciantes conozcan sobre el cumplimiento de obligaciones fiscales. El problema abordado fue: ¿Cómo una metodología tributaria incide en la mejora de obligaciones fiscales en asociaciones de ámbito socioeconómico? Se determinaron las necesidades de información para acoger medidas que permitan visualizar herramientas que contribuyan al conocimiento y cumplimiento de tributos. La propuesta nace del diagnóstico sistemático a partir de una investigación basada en una sistematización bibliográfica de alta rigurosidad articulado con análisis bibliométrico, con el propósito de precisar elementos que connotan el paradigma estudiado. Asimismo, otros métodos adoptados fueron análisis documental, utilizado para la valoración de bibliografía relacionada con el problema; histórico lógico para establecer fundamentos asociados a la cultura tributaria; análisis y síntesis que proporcionó información con base más ajustada para abordar el problema científico; inductivo-deductivo que accedió al análisis de antecedentes particulares de los problemas existentes. Los resultados permitieron identificar la necesidad de desarrollar una propuesta metodológica que contribuya a la mejora de obligaciones fiscales en asociaciones de ámbito socioeconómico. Además, se logró determinar que el tema investigado no ha sido aprovechando en su totalidad en diferentes universidades de Ecuador, por lo que se considera viable realizar propuestas enfocadas a la mejora de la cultura tributaria.

PALABRAS CLAVE: Tributos; Obligaciones fiscales; Cumplimiento tributario; Cultura Tributaria.

\footnotetext{
${ }^{1}$ Doctor en Ciencias Económicas, docente de la carrera de Contabilidad y Auditoría de la Facultad de Ciencias Económicas, Universidad Estatal del Sur de Manabí, Ecuador. Orcid-0000-0001-9798-9780. E-mail: mantawilliam@gmail.com

${ }^{2}$ Estudiante de noveno semestre de la Carrera de Contabilidad y Auditoría de la Facultad de Ciencias Económicas, Universidad Estatal del Sur de Manabí, Ecuador. E-mail: alay-tamara2163@unesum.edu.ec
} 
William Patricio Proaño Ponce, Tamara Valeria Alay Barcia

\title{
ANALYSIS OF FISCAL OBLIGATIONS ESTABLISHED FOR SOCIO-ECONOMIC ASSOCIATIONS IN JIPIJAPA, MANABÍ
}

\begin{abstract}
At present, complying with tribute duties has become a right for every society. However, many socio-economic associations in Ecuador are not fully informed about the rights that correspond as such. Among the factors are: misinformation, meager tribute culture. That is why, it is essential that merchants know about compliance with fiscal obligations. The problem addressed was: how a tribute methodology affects the improvement of tribute obligations in socio-economic associations. Information needs were determined to accommodate measures that allow visualizing tools that contribute to knowledge and compliance with tributes. The proposal arises from a systematic diagnosis based on a research based on a highly rigorous bibliographic systematization articulated with bibliometric analysis, in order to specify elements that connote the studied paradigm. Likewise, other methods adopted were documentary analysis, used for the evaluation of bibliography related to the problem; logical history to establish foundations associated with the tribute culture; analysis and synthesis that provided information with a more accurate basis to address the scientific problem; inductive-deductive that accessed the analysis of particular antecedents of the existing problems. The results made it possible to identify the need to develop a methodological proposal that contributes to the improvement of fiscal obligations in socio-economic associations. In addition, it was possible to determine that the subject investigated has not been fully exploited in different universities in Ecuador, therefore, it is evident to make proposals focused on the improvement of the tribute culture.
\end{abstract}

KEYWORDS: Tributes; Fiscal obligations; Tribute compliance; Tribute culture.

\section{INTRODUCCIÓN}

Hoy en día se observa de manera invariable como en una economía globalizada las asociaciones atraviesan por problemas en el cumplimiento de las obligaciones fiscales con incidencia en su liquidez. En este contexto, Castillo (2015), expresa que la mayoría de países deben buscar un equilibrio tributario que conlleve a una sostenibilidad de los negocios. Por lo que, al existir una relación muy estrecha entre las políticas fiscales y la administración de los negocios se debe garantizar una estabilidad económica para el buen desempeño local y nacional.

Las aportaciones de la Comisión Económica para América Latina y el Caribe (CEPAL, 2018), establecen que el elevado nivel de evasión, morosidad y elusión constituye un factor que favorece a explicar el exiguo desempeño de la cultura tributaria. Además, los estudios de Orellana, Gutiérrez, y Feijoo (2019), muestran aptitudes existentes en temas de fiscalización donde diversos gobiernos han establecido convenios favoreciendo a negocios en tratos tributarios para contribuyentes con el fin de erradicar esta conducta.

En este contexto, es importante acoger los aportes de Lucio y Sornoza (2017), quienes exponen que en Ecuador el incumplimiento de normativas fiscales conlleva al empleo de sanciones las mismas que transgreden en la economía e imagen de entidades. En este sentido, se considera que

68 UNESUM-Ciencias. Publicación cuatrimestral. Vol. 4, No. 4 (Septiembre-Diciembre), Año 2020. 
la política fiscal no debe diseñarse sin tener en cuenta la naturaleza de las asociaciones debido a que esta y tipo de choque que enfrenta una economía.

Con relación a lo mencionado Proaño, Lucio y Yoza (2020), establecen que diversos negocios en Manabí, Ecuador no crecen debido a que sus dueños no se capacitan o no tienen desarrollada una cultura tributaria que permita un correcto proceso a trámites fiscales. Asimismo, establecen que en estos negocios los propietarios aseguran ser autosuficientes y otros, tienen miedo acrecer donde la problemática en las actividades comerciales se ve combinada con una mirada desde lo externo (economía, política, tecnología) y de lo interno (mercadeo, producción, personal).

Además, se debe precisar que la evasión tributaria es una de las obligaciones fiscales que se encuentra enmarcada como una práctica adoptada en la sociedad donde sus orígenes tienden a variar de acuerdo a su lugar de procedencia. Igualmente, (Collosa, 2019) expone diferentes razones por la que surge la elusión fiscal; como la estructura propia del sistema tributario, falta de simplicidad y precisión de la legislación tributaria, inflación y demás que influyen en la evasión y que genere efectos adversos en la estabilidad económica.

En otro orden de ideas, se debe precisar a la cultura tributaria como un conjunto de valores y comportamientos acogido en la sociedad, que fusiona como una conducta manifestada en el cumplimiento permanente de los deberes tributarios y responsabilidad ciudadana. En este contexto, la Universidad Estatal del Sur de Manabí (UNESUM, 2020), en estudios realizados en este campo, establece la necesidad del conocimiento en las principales leyes tributarias, las que en ocasiones diversos contribuyentes las desconocen, al igual considera oportuno el conocimiento de los deberes que poseen los contribuyentes, los cuales de una u otra manera se catalogan como responsables de las obligaciones tributarias.

Con relación a las asociaciones del ámbito socioeconómico propósito del presente estudio, se debe precisar que estas se clasifican de acuerdo a características propias mediante el desenvolvimiento en que se desarrollen. En este sentido, la Asamblea Nacional (2018), las define como un conjunto de personas que se han unido en forma voluntaria para satisfacer sus necesidades económicas, sociales y culturales en común, mediante una gestión dinámica. Es este contexto, se reconoce a diversas asociaciones de este ámbito como unidades económicas populares que nacieron en medio de la lucha por la generación de ingresos y sobrevivencia de socios que crearon su propia actividad económica como mecanismo de supervivencia.

Se considera que estas asociaciones se consolidan con base al apoyo mutuo entre socios, donde el intercambio de conocimientos, mercado son estrategias que fortalecen el factor solidario y con ello logran satisfacer las necesidades básicas del grupo donde los procesos se desarrollan desde lo local. Esta lógica permite inclusive generar emprendimientos en un espacio de mercadeo más competitivo impulsando el desarrollo de abajo hacia arriba.

En otro orden de ideas y con relación a las metodologías tributarias, estas dependen de la necesidad por la que esté pasando la asociación de ámbito socioeconómico. En este contexto Salas (2010), refiere como estas contribuyen para el registro correcto de la información financiera con el fin de facilitar la determinación de impuestos a declarar ante la administración tributaria. 
Asimismo, Bustamante y Moncayo (2011), exponen medidas incentivadoras y preventivas enfocadas al cumplimiento tributario, estas permiten determinar que las metodologías deben estar integradas por un conjunto de estrategias que contribuyan a la mejora de obligaciones fiscales.

De lo antedicho, se debe considerar que una gestión administrativa se aprecia por el cumplimiento de sus metas y de su operatividad, las cuales deben responder a las políticas fiscales. En este sentido, Bernal (2020), muestra algunos procedimientos contables, financieros y administrativos enfocados a mejorar la gestión tributaria en la administración. En este sentido, se considera que una correcta planificación llevada a través de una metodología, proveerá a las asociaciones el usar estrategias que, amparadas en la normativa tributaria vigente, permita a este grupo económico cumplir con las obligaciones tributarias establecidas.

Es por ello que el presente artículo persigue como propósito: diseñar una metodología tributaria para la mejora de obligaciones fiscales en asociaciones de ámbito socioeconómico del cantón Jipijapa, de esta manera contribuya a la mejora en obligaciones fiscales mediante una cultura tributaria y conciencia colectiva adecuada

\section{DESARROLLO}

Para verificar el propósito investigativo, se logró una trayectoria metodológica la cual abordó un reconocimiento bibliográfico que se constituyó en etapa fundamental y garantizó la obtención de información más relevante en el campo de estudio. Además, el método de medición se empleó en el proceso de generación de conocimiento como actividad fundamental que buscó la observación de aspectos de la realidad, donde la clasificación de los ítems en el constructo de los temas investigados según su previa definición y evaluación de su grado de relevancia.

Se aplicó búsqueda especializada mediante análisis bibliométrico con la finalidad de conocer indicadores que facultaran la descripción en el uso de metodologías tributarias para la mejora de obligaciones fiscales que contribuya a fortalecer la presente investigación y construcción de una metodología en este campo con miras de repensar la cultura de investigación en el área de la cultura tributaria. En este contexto, es importante acoger los aportes de Cortés (2007), quien determina que la bibliometría es reconocida como: “conjunto de conocimientos metodológicos aplicados a la medida, a través de indicadores, del número de documentos publicados y de las citas que estos mismos documentos reciben, de acuerdo con su origen geográfico” (p.44). En este contexto, se considera que este procedimiento contribuye a la evaluación de los productos de la ciencia.

La utilización de fuentes de información secundaria permitió determinar los antecedentes y las tendencias actuales vinculadas al objeto de estudio. El método inductivo-deductivo, accedió al análisis de antecedentes particulares de los problemas existentes en las asociaciones de ámbito socioeconómico y su rol en las obligaciones fiscales. Además, el método analítico- sintético, permitió detallar elementos del fenómeno estudiado.

\section{RESULTADO Y DISCUSIÓN}

70 UNESUM-Ciencias. Publicación cuatrimestral. Vol. 4, No. 4 (Septiembre-Diciembre), Año 2020. 
La presente investigación fue elaborada en el año 2020 con el propósito de analizar el cumplimiento de las obligaciones fiscales de los contribuyentes que conforman las asociaciones de ámbito socioeconómico del cantón Jipijapa, mediante fuentes bibliográficas que permitieron conocer resultados de estrategias aplicadas en una metodología tributaria. Se efectuó en la carrera de Contabilidad y Auditoría de la Universidad Estatal del Sur de Manabí.

Con el objeto de analizar cómo se distribuye la deliberación en informes de tesis en el área de la cultura tributaria, se consideraron trabajos presentados entre el año 1990 y 2020 en 10 universidades del Ecuador. Inicialmente se analizarían 100 tesis. No obstante, se realizó el estudio solo con 50 informes de tercer nivel, debido a que solo estas realizaban estudios enfocados a los propósitos del presente estudio. Asimismo, muchas están en los repositorios de las universidades al estar en trámite de digitalización y subida de información. Adicionalmente, a efectos directos que sufren los investigadores debido a la catástrofe del coronavirus SARS-CoV2, como es el no poder ir de manera directa a las salas de tesis de las universidades por estar cerradas.

La recolección de información a partir de los ejemplares de las tesis investigadas permitió organizar cada informe por año de publicación (tabla 1). Al realizar el análisis se dedujeron los rasgos esenciales de cada una de ellas, como el desarrollo del contenido para llevar a cabo cada propuesta, de esta manera se pudo conocer cada propósito establecido y la inclinación de cada tema desarrollado. Los principales resultados muestran que desde el año 2015 a la fecha es donde se ha dado mayor importancia desde la ciencia para investigar la cultura tributaria lo que corresponde a un $70 \% \%$ de los informes clasificados como originales, de reflexión al tema de estudio. No obstante, y sin ser menos importante, se debe destacar que desde el año 1990 con un 2\% exponen resultados, en el año 1994 los aportes fueron del 4\%, en el año 1998 con el 6\% y de manera progresiva ya en el 2010 se visualiza un 10\%, a pesar que en el 2011 solo un 8\% de informes acogían el interés respecto a la necesidad de una metodología tributaria para la mejora de obligaciones fiscales.

Tabla 2. Tesis por año

\begin{tabular}{|c|c|}
\hline Año publicación & Porciento de tesis por año \\
\hline 1990 & $2 \%$ \\
\hline 1994 & $4 \%$ \\
\hline 1998 & $6 \%$ \\
\hline 2010 & $10 \%$ \\
\hline 2011 & $8 \%$ \\
\hline 2015 & $14 \%$ \\
\hline 2018 & $12 \%$ \\
\hline 2019 & $20 \%$ \\
\hline 2020 & $24 \%$ \\
\hline TOTAL & $100 \%$ \\
\hline \multicolumn{2}{|c|}{ Fuente: elaboración propia } \\
\hline
\end{tabular}


Respecto a los métodos utilizados en las diferentes tesis, se observaron concepciones múltiples que permitieron tener diversos criterios del fenómeno investigado y generar confusión. Es así qué, la síntesis, análisis documental, comparativo, de campo, lo declaran un 8,33\% de las investigaciones. Por otro lado, el método de análisis descriptivo, inductivo, deductivo fueron aprovechados en un $12,50 \%$. En su mayoría las tesis investigadas trabajaron con casos prácticos esto es un $20,83 \%$, es un aporte significativo que contribuye a la presente investigación. Los métodos estadísticos, así como explicativo, fueron utilizados en un 4,17\%. Se considera que este bajo porcentaje no da carácter estadístico para apreciar resultados. En la figura 1 se aprecia lo detallado para un análisis más gráfico.

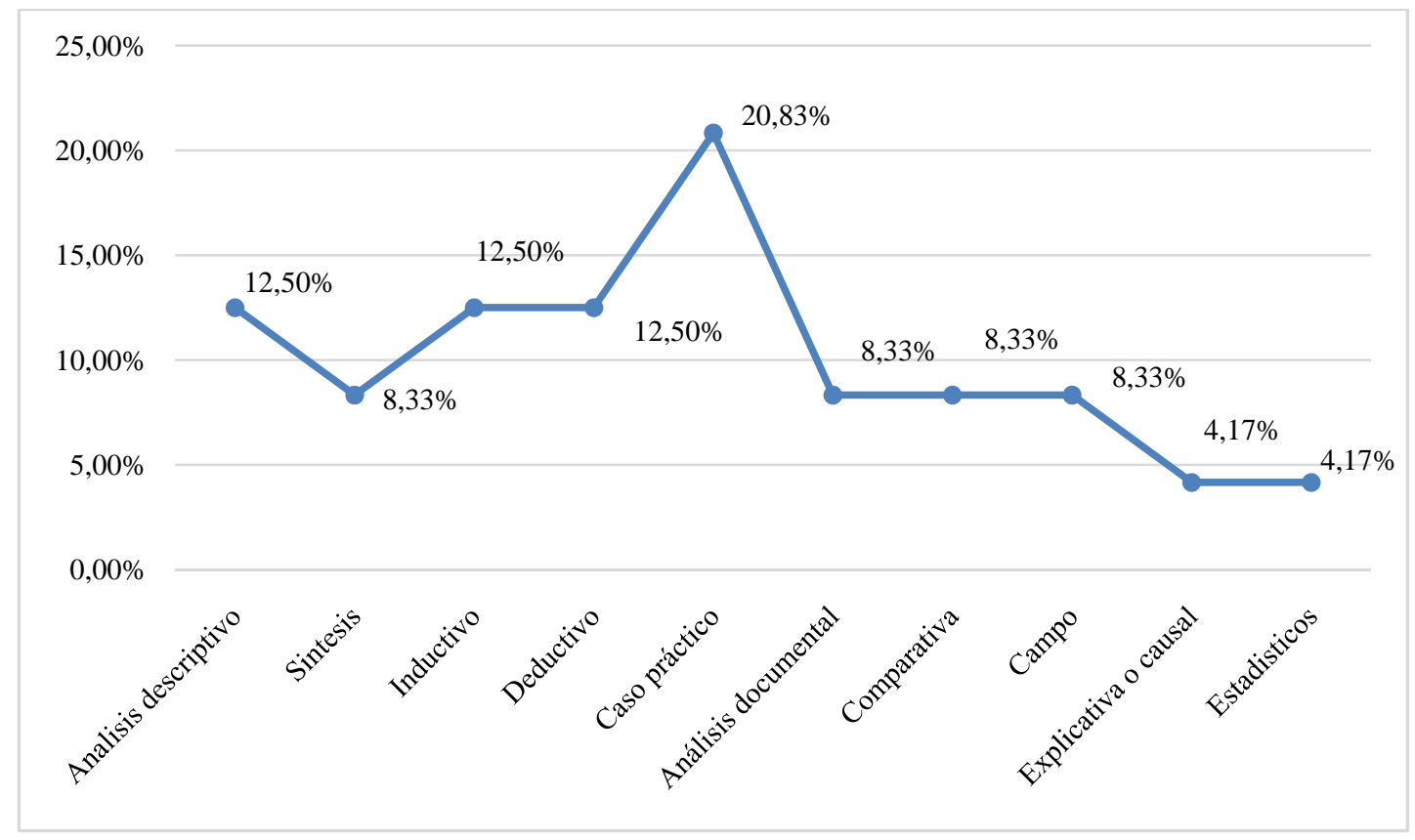

Figura 1. Métodos utilizados en tesis investigadas Fuente: elaboración propia

Con relación a los autores tanto personales como corporativos, se analizó los más registrados, los cuales fueron 440 como se muestra en la tabla. 2. Se tomaron referencias cuya frecuencia sea superior o igual a 4 respecto a las citas del conjunto de material. Se puede apreciar como los documentos del Servicio de Rentas Internas (SRI), son los más mencionados con un 39,55\% de ocurrencias. El índice de colaboración de la Ley Orgánica de Régimen Tributario (LORT), estuvo en un 21,59\%, la cual contribuyó en fundamentos teóricos de la presente investigación. El Reglamento a la Ley de RUC denota un 9,77\% en investigación. Por otro lado, el Código Orgánico Tributario, contribuyó con una relación del 7,95\% en el desarrollo de la información en los informes analizados.

De manera más detallada en la tabla 2 muestra el comportamiento de los investigadores hacia las referencias más aprovechadas en el campo de estudio de la tesis. Las mencionadas en anterioridad son fuentes que cuentan con una mayor frecuencia para llevar a cabo el desarrollo de 
la fundamentación teórica. Siendo las de más contenido relevante para la investigación llevada a cabo.

Tabla 2. Referencias más examinadas

\begin{tabular}{|l|r|r|}
\hline AUTORES & FRECUENCIA & PORCENTAJE \\
\hline Asamblea Constituyente & 7 & $1,59 \%$ \\
\hline Servicio de Rentas Internas & 174 & $39,55 \%$ \\
\hline Ley Orgánica de Régimen Tributario Interno & 95 & $21,59 \%$ \\
\hline Ministerio de Finanzas & 13 & $2,95 \%$ \\
\hline Centro Interamericano de Administraciones Tributarias & 5 & $1,14 \%$ \\
\hline Código Orgánico Tributario & 35 & $7,95 \%$ \\
\hline INEC & 22 & $5,00 \%$ \\
\hline Contraloría General del Estado. & 3 & $0,68 \%$ \\
\hline Comisión Económica para América Latina (CEPAL) & 12 & $2,73 \%$ \\
\hline Reglamento a la Ley de RUC & 43 & $9,77 \%$ \\
\hline Acebedo Rubén & 4 & $0,91 \%$ \\
\hline García Ríos, J. L. & 5 & $1,14 \%$ \\
\hline Jorrat de Luis, M. & 5 & $1,14 \%$ \\
\hline Carrasco, C. M. & 8 & $1,82 \%$ \\
\hline Rivas, Norberto, and Samuel Vergara. & 4 & $0,91 \%$ \\
\hline Roca, J. & 5 & $1,14 \%$ \\
\hline TOTAL & 440 & $100,00 \%$ \\
\hline
\end{tabular}

Fuente: elaboración propia

Del análisis de informes de tesis, se considera que estas son fuente que permiten obtener información del conjunto de tendencias de la cultura tributaria que han sido examinados en un determinado momento por investigadores de pregrado que ahora son profesionales en el campo amplio de la contabilidad y auditoría. Estos estudios permitieron definir estrategias a seguir en el estudio y creación de una propuesta que acceda al diseño de una metodología apropiada para la sociedad y que esta contribuya al desarrollo de la cultura tributaria en asociaciones de ámbito socioeconómico como lo es el propósito de este estudio.

En la trayectoria de análisis establecido en las tesis, fue evidente apreciar como cada investigador ejerció una metodología favorable a su institución. Con relación a lo mencionado Soto (2018), analiza la independencia que tiene cada universidad en momentos de plasmar sus estrategias. No obstante, expone que la academia en Ecuador aún está en deuda con la sociedad las cuales deben ejercer su rol protagónico y actuar sobre la manera de comprender a la sociedad. Se considera que esto contribuirá el entrever limitaciones cuando se desee medir la investigación y con esto crear intercambios inter-universidades a través de redes personales o por carrera para el manejo de fuentes de información como mecanismos de articulación. 
En otro orden de ideas, es preciso exponer la existencia de dificultades para realizar investigaciones a nivel de pregrado. En este contexto, la Universidad Central del Ecuador (UCE, 2018), establece la existencia de desarticulación entre instancias académicas que realizan actividades de investigación. Sumado a esto, se considera la desactualización de colecciones en la biblioteca de la universidad, exigua plataforma tecnológica. Factores que inciden en la variedad y tipos de fuentes consultadas al momento de elaborar la investigación.

Son significativos los aportes de Herrera-Galán y Duany-Alfonzo (2016), quienes determinan que la puesta en marcha de una metodología adecuada a una organización permitirá implementar procesos con soluciones prácticas y de fácil implementación. En este contexto, el diseñar una metodología tributaria para la mejora de obligaciones fiscales en asociaciones de ámbito socioeconómico del cantón Jipijapa, favorecerá de mejor manera la toma de decisiones en los líderes y actores involucrados en organizaciones del tipo estudiado.

La implementación de una metodología constituye una gran oportunidad para el desarrollo de la cultura tributaria en asociaciones de ámbito socioeconómico, debido a su vinculación y articulación con el entorno, obligaciones fiscales, capacitaciones y calendario tributario. Que supone a la vez se coyuntura de una infraestructura de soporte tributario/tecnológico apropiada para alanzar las opciones escogidas y se convierta en una herramienta de mejora en las obligaciones fiscales enfocada a los actores locales del cantón jipijapa.

- De manera fundamental la metodología tributaria está diseñada para resolver procesos complejos a través de fases integrales y participación ciudadana con roles y responsabilidades, por lo que se considera como meta el bienestar de los socios. Asimismo, corresponde el desarrollo de una cultura tributaria que caracterice a este grupo social.

- La metodología tributaria involucra a actores de mayor influencia en la gestión de procesos tributarios que tengan las asociaciones de ámbito socioeconómico como: Servicio de rentas Internas (SRI), Núcleo de Apoyo Financiero (NAF) de la Universidad Estatal del Sur de Manabí, sector comunitario de los socios.

- El análisis comparativo entre diferentes estudios de metodologías tributarias beneficio un soporte teórico a la metodología propuesta, más allá del contexto nacional en el cual se basan sus condiciones de implementación.

- Para la metodología, la conformación del equipo líder, se constituye en el centro decisor primordial al ser quienes analizan los distintos procesos tributarios que solventará la asociación, tendrá las competencias para planificar el desarrollo de la cultura tributaria y formular lo correspondiente con los planes para la ejecución de las obligaciones fiscales de manera articulada la planificación del Servicio de Rentas Internas (SRI).

- La propuesta metodológica se cimienta con los lineamientos y estrategias impulsados por la Secretaria Nacional de Planificación y Desarrollo (SEMPLADES, 2019), a través de la Agenda Zonal 4, donde se estipula la necesidad de elaborar procesos metodológicos que 
permitan de manera integral la mejora en la prestación de servicios públicos y que garanticen la calidad, accesibilidad, pertinencia.

En este contexto, y para el desarrollo de la cultura tributaria, los autores consideran fortalecer factores como: la formalización y promoción de negocios, simplificación administrativa, conseguir objetivos económicos y sociales, que apunten a una mejor calidad de vida de la población en general.

La Metodología tributaria para la mejora de obligaciones fiscales en asociaciones de ámbito socioeconómico del cantón Jipijapa desde su entorno parte de principios, leyes y normas que tienen las obligaciones fiscales, esto garantizará la continuidad de cada una de las fases. Además, la metodología contempla la conformación de un equipo líder con roles y responsabilidades que denotan el impulso a la cultura tributaria de los socios como actores principales del presente estudio. La infraestructura de soporte tributario/tecnológico, permitirá retroalimentar los planes de capacitación de pago de impuestos y evasión fiscal, así como generar eficacia y oportunidad de las estrategias y contribuir en fallas de control.

Además, la metodología facilita la aplicabilidad en la práctica social de las asociaciones de ámbito socioeconómico. La representación gráfica muestra tres fases: valoración tributaria, valoración del cumplimiento de obligaciones fiscales, respuesta a la evasión. Las mismas están conformadas por pasos que se muestra en la figura 2. 


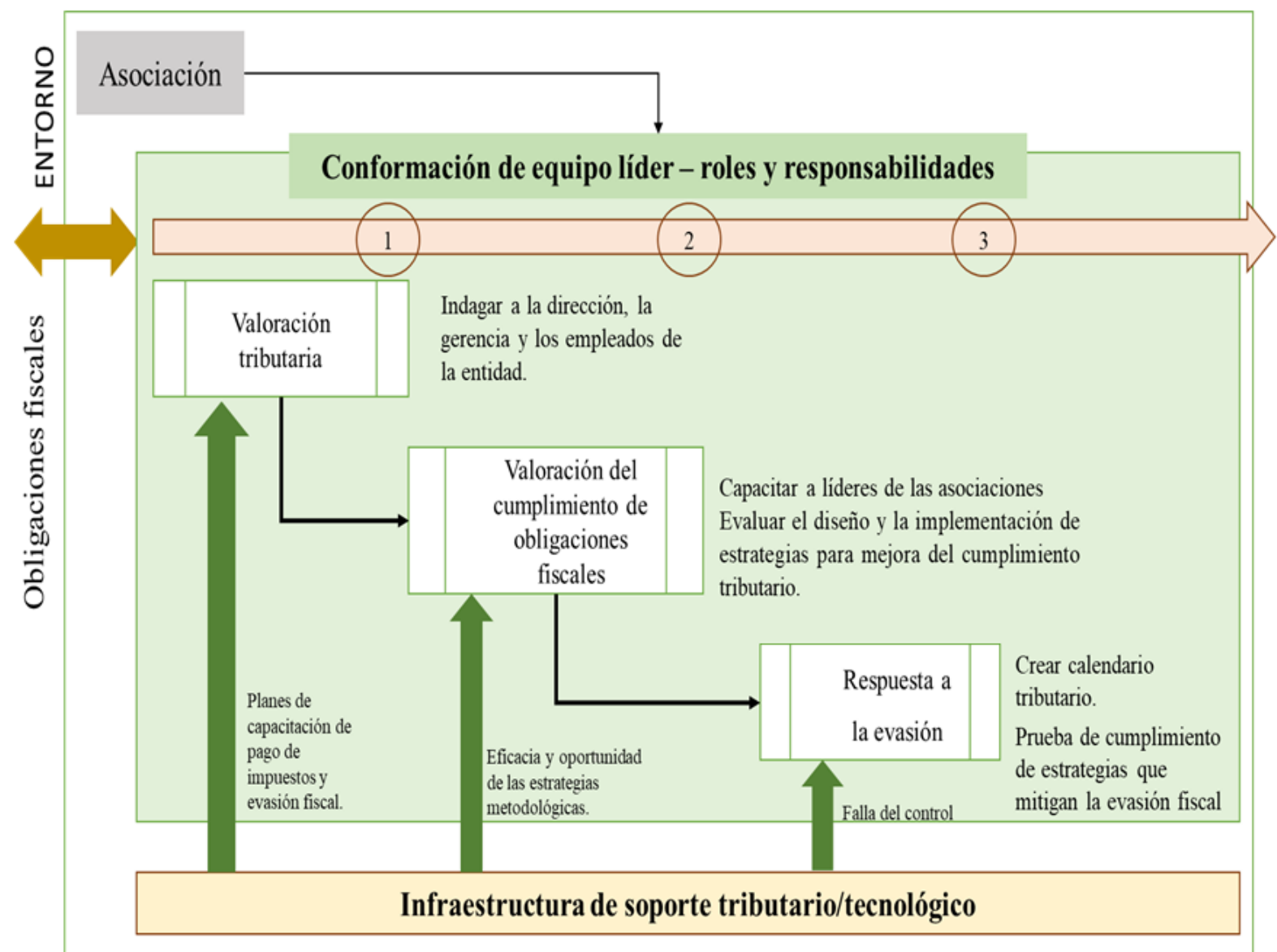

Figura 2. Metodología tributaria para la mejora de obligaciones fiscales en asociaciones de ámbito socioeconómico del cantón Jipijapa (MTMOFAASCJ)

\section{CONCLUSIONES}

Fuente: Elaboración propia

A partir de la fundamentación teórica y metodológica realizada, los resultados de la investigación mostraron brechas relacionadas con el desarrollo de la cultura tributaria enfocada a la mejora de las obligaciones fiscales. Contribuyendo a la esquematización de una metodología tributaria en asociaciones de ámbito socioeconómico del cantón Jipijapa que permite la mejora de obligaciones fiscales de este grupo económico, así como optimizar la toma de decisiones de los líderes locales involucrados en el desarrollo de la cultura tributaria.

El diagnóstico realizado a través de una sistematización bibliográfica de alta rigurosidad que con la utilización de análisis bibliométrico de literatura en diferentes universidades, permitió identificar problemáticas en metodologías tributarias, donde pocas describen procedimientos contables, financieros y administrativos que contribuyan a la mejora de la gestión tributaria en la administración de asociaciones. 
A partir de los resultados de la presente investigación, se detectaron brechas relacionadas con la cultura tributaria, esto contribuye a la concepción futura de un modelo para el desarrollo de la cultura tributaria en asociaciones de ámbito socioeconómico, en función del aprovechamiento de los recursos endógenos y exógenos y la articulación de actores locales que permitirá mejorar las obligaciones fiscales y contribuir en la toma de decisiones a los líderes en el desarrollo de las asociaciones involucradas.

\section{REFERENCIAS BIBLIOGRÁFICAS}

Asamblea Nacional. (2018). Ley Orgánica de la Economía Popular y Solidaria y del Sector Financiero Popular y Solidario. Subsecretaria Nacional de la Administración Pública. Quito, Ecuador: Registro Oficial 444, modificación: 23-oct.-2018.

Bernal Bernal, R. (2020). Propuesta metodológica para mejorar la gestión tributaria en la administración de las PYMES florícolas en la provincia de Pichincha cantón Quito. Universidad Politécnica Salesiana de Ecuador, Posgrado. Quito, Ecuador: UPSE.

Bustamante P., W., \& Moncayo E., C. (2011). Diseño e implementación de estrategias para la consolidación de una efectiva cultura tributaria en el sector comercial de la bahía de Guayaquil "Cooperativa 4 de Agosto". Universidad Politécnica Salesiana de Ecuador, Unidad de Posgrados. Guayaquil-Ecuador: UPSE.

Castillo Bueno, E. L. (2015). Análisis de las obligaciones fiscales y su incidencia en la liquidez de la asociación de mariscadores autónomos y afines de Machala. El Oro, Ecuador: UTM.

Collosa, A. (2019). Evasión Tributaria en América Latina: Un Llamado de atención para todos. CIAT. Centro Interamericano de Administraciones Tributarias. CIAT.

Comisión Económica para América Latina y el Caribe (CEPAL). (2018). Panorama Fiscal de América Latina y el Caribe. Los desafíos de las políticas públicas en el marco de la Agenda 2030. Publicación de las Naciones Unidas ISBN: 978-92-1-121982-1, División de Desarrollo Económico de la CEPAL. CEPAL.

Córdoba, M. E. (2016). Reflexión sobre la formación investigativa de los estudiantes de pregrado. Revista Virtual Universidad Católica del Norte. ISSN: 0124-5821( núm. 47), pp. 20-37. Obtenido de https://www.redalyc.org/articulo.oa?id=1942/194244221003

Cortés Vargas, D. (2007). Medir la producción científica de los investigadores universitarios: bibliometría sin límites. Revista de la Educación Superior. ISSN: 0185-2760., Vol. XXXVI (2)(No. 142), pp. 43-65. Obtenido de http://www.scielo.org.mx/pdf/resu/v36n142/v36n142a3.pdf

Herrera-Galán, M., \& Duany-Alfonzo, Y. (2016). Metodología e implementación de un programa de gestión de mantenimiento. Ingeniería Industrial. ISSN 1815-5936, vol.37 (no.1), 2-13. Obtenido de http://scielo.sld.cu/scielo.php?script=sci_arttext\&pid=S1815-59362016000100002

Lucio Pillasagua, A., \& Sornoza Parrales, D. (2017). LA auditoría tributaria y el cumplimiento de las obligaciones tributarias en instituciones de la iglesia católica con actividades de educación. UNESUM-Ciencias: Revista Científica Multidisciplinaria. ISSN: 2602-8166, Vol. 1(Núm. 3), 15-35. doi:https://doi.org/10.47230/unesum-ciencias.v1.n3.2017.24

Orellana, M. N., Gutiérrez, N. D., \& Feijoo, E. M. (2019). Los convenios de doble tributación en la recaudación de la administración tributaria en Ecuador. Revista ESPACIOS. ISSN: 0798 1015, Vol. 40(N³8), pág. 10.

Proaño Ponce, W., Lucio Pillasagua, A., \& Yoza Rodríguez, N. (2020). Contabilidad bajo la perspectiva de dispositivos electrónicos en contribuyentes de microempresas de Jipijapa, Manabí. Avances. ISSN: $1562-$ 3297, Vól. 22( $\left.\mathrm{N}^{\circ} 2\right)$, p. 222-238. Obtenido de http://www.ciget.pinar.cu/ojs/index.php/publicaciones/article/view/536 
Salas Suárez, M. (2010). Diseño e implementación de una metodología para el control tributario e impuestos de personas naturales obligadas a llevar contabilidad. Universidad Politécnica Salesiana- Sede Quito. Quito, Ecuador: UPS-SQ.

Secretaria Nacional de Planificación y Desarrollo (SEMPLADES). (2019). Agendas Zonales. Zona 4 PacíficoManabí y Santo Domingo de los Tsáchilas 2017-2021. Subsecretaría General de Planificación y Desarrollo, Subsecretaría de Planificación Territorial, Dirección de Planificación Zonal 4 . Manta, Ecuador: (C) Senplades.

Soto Dávila, O. (2018). La Responsabilidad Social en la Universidad Ecuatoriana. Razón y Palabra. Primera Revista Electrónica en Iberoamérica Especializada en Comunicación. ISSN:1605-4806, Vol.22(3_102), pp. 340357.

Universidad Central del Ecuador, UCE. (2018). Plan Estratégico de Desarrollo Institucional de la Universidad Central del Ecuador. Universidad Central del Ecuador. Quito, Ecuador: UCE.

Universidad Estatal del Sur de Manabí. UNESUM. (2020). Estrategias para el Desarrollo de la Cultura Tributaria en Escenarios Permanentes de la UNESUM - cantón Jipijapa. Proyecto de Investigación Científica, Desarrollo Tecnológico e Innovación (I+D+i). Jipijapa, Ecuador: UNESUM. 